\title{
El aprendizaje organizacional, prueba piloto de instrumentos tipo Likert
}

\author{
Manuel Alfonso Garzón Castrillón / castrillonm@uninorte@edu.co \\ Universidad del Norte \\ Barranquilla, Colombia
}

André Luis Fischer / afischer@usp.br

Universidad de Sao Paulo

Sao Paulo, Brasil

\section{RESUMEN:}

Este artículo presenta los resultados obtenidos en la prueba piloto de instrumentos construidos para cumplir con el objetivo específico de diseñar y convalidar instrumentos que permitan caracterizar la capacidad de aprendizaje organizacional. La escala de medición utilizada fue tipo Likert, la cual permitió establecer la pertinencia de la dimensión como variable de análisis en el contexto del aprendizaje organizacional. Se utilizó el procedimiento de Análisis de Varianza -ANOVA- a una vía, con el paquete estadístico SPSS. ${ }^{1}$ Se simplificaron 138 variables en 3 factores y 40 afirmaciones.

Palabras clave: aprendizaje organizacional, modelos teóricos, capacidad de aprendizaje.

\section{ABSTRACT:}

This paper presents the results obtained in the pilot study of instruments created to comply the specific objective of designing and validating instruments to study the capacity of organizational learning. The Likert measurement scale was used because it allowed to establish the pertinence of the dimension as variable in the context of organizational learning. A One-way Analysis of Variance (ANOVA) was used, with statistical package SPSS. Some 138 variables in 3 factors and 40 affirmations were simplified.

Keywords: organizational learning, theoretical models, capacity of learning.

\footnotetext{
${ }^{1}$ Statistical Package for the Social Science 15.00.
} 


\section{INTRODUCCIÓN}

El estudio sobre el aprendizaje organizacional se inició en 1963 con la obra de Richard Cyert y James March, quienes presentaron cinco puntos claves sobre su teoría de la elección y control organizacional. Dos años después aparece el término aprendizaje organizacional, en una publicación de Miller y Cangelloti (1965), basándose en la Teoría de Contingencias ${ }^{2}$, quienes propusieron el modelo conceptual "adaptación-aprendizaje", para explicar el porqué sólo algunas instituciones sobreviven a las exigencias de sus entornos a través del tiempo.

Aunque ya Follet et al. (1960), habían afirmado que las personas en las organizaciones pueden coordinar, ordenar y sistematizar el conocimiento con un gran aporte a la concepción de la organización y a la cooperación de sus componentes.

En este orden de ideas, a pesar de tener claro el origen del concepto, el primer obstáculo al que se enfrenta el investigador sobre aprendizaje organizacional es que la literatura sobre aprendizaje organizacional ha crecido en los últimos tiempos, no solamente en volumen, sino también, descoordinado las dimensiones del tema. La disciplina se sitúa en la confluencia de diferentes campos de investigación de forma interdisciplinaria, como lo afirma Dogson (1993): la psicología, la sociología, la economía y la administración.

De la misma forma, la capacidad de aprendizaje ha sido considerada y valorada como una variable multidimensional. A partir de estos planteamientos, la capacidad de aprendizaje de una organización está determinada por cuatro variables fundamentales (Garzón, 2008): las fuentes, los niveles de aprendizaje, la cultu-

\footnotetext{
${ }^{2}$ Las investigaciones pioneras son las de la socióloga inglesa Joan Woodward (1965) quien a finales de los años cincuenta estudió cien firmas industriales en la región de South Essex, enfocándose en la relación entre tecnología y estructura organizacional, el contingente o situacional sostiene que la estructura organizacional y el sistema administrativo dependen o son contingentes respecto de factores del medio ambiente, de la organización, la tarea y la tecnología. No existe entonces una estructura organizacional óptima que sirva para todas las circunstancias, lo cual contradice lo que había sido un supuesto central de cada teoría (Desler, 1976).
} 
ra y las condiciones para de aprendizaje. Según Bontis (1999) y Vera y Crossan (2000) estas variables se pueden caracterizar en dos dimensiones:

1) Una dimensión estática, constituida por contener reservas de conocimiento (Stocks of knowledge's), inmersos en la organización y que residen en los individuos, los grupos y en la propia organización; y

2) Una dimensión dinámica, determinada por los procesos de aprendizaje que hacen posible la evolución de las reservas (Stocks of knowledge's), por medio de la activación de "flujos" de generación, absorción, difusión y utilización del conocimiento. Así, los almacenes de conocimiento son la entrada y salida de un conjunto de flujos o procesos de aprendizaje que, en la terminología de March (1991), hacen posible la exploración y la explotación de los conocimientos.

En definitiva, la interacción dinámica entre las fuentes, los sujetos, la cultura y las condiciones para el aprendizaje, los conocimientos y los procesos de aprendizaje que los desarrollan, determinan la capacidad de aprendizaje de las organizaciones, cuyos efectos sobre los resultados de la organización son modelados por la gestión del conocimiento.

Con estas premisas, en este trabajo de investigación se plantea la pregunta: ¿la capacidad de aprendizaje con las fuentes, los sujetos, la cultura y las condiciones para el aprendizaje son determinantes en los resultados organizacionales?

Como justificación se propone que la respuesta al problema de investigación planteado requiere el estudio de los factores internos y externos que inciden en el aprendizaje organizacional, debido a que este nuevo enfoque no está claramente establecido en las organizaciones. Teniendo en cuenta que la situación actual de las organizaciones se caracteriza por recursos disminuidos, que en lugar de ser una amenaza son una oportunidad para promover ambientes de aprendizaje en donde el diálogo es la herramienta que agrega valor, con personas dispuestas a compartir conocimientos. 
Por lo tanto, ocuparse del aprendizaje organizacional tiene sentido por los siguientes motivos:

- La tendencia actual que con más fuerza está cambiando a las organizaciones es la creación y valoración del conocimiento.

- El conocimiento encarnado en las personas es decisivo para el desarrollo económico y la productividad.

- El incremento de las capacidades de los empleados, la promoción y el fomento del aprendizaje organizacional son armas estratégicas al servicio de las organizaciones.

- El capital humano ha adquirido protagonismo como fuente de diferenciación de una organización frente a otra pues el conocimiento de las personas hace la diferencia.

El proyecto es válido en la perspectiva de los objetivos planteados, porque permitió:

- Validar los instrumentos diseñados para caracterizar la capacidad de aprendizaje organizacional orientados a determinar cómo influyen sobre los resultados de la organización.

- Proporcionar instrumentos confiables para determinar la incidencia de los factores que determinan las fuentes, los niveles, la cultura y las condiciones para el aprendizaje, en el contexto del marco teórico elaborado por el proyecto y su impacto en el aprendizaje organizacional.

- Generar conocimiento para el estudio del aprendizaje organizacional y su influencia sobre los resultados de la organización y la incidencia en la sostenibilidad de las organizaciones.

\section{MARCo teóRICO}

El marco teórico se basa en la propuesta de modelo de aprendizaje organizacional de Garzón (2008) quien establece que la primera variable se denomina fuentes; la segunda variable, sujetos; la 
tercera, cultura y la cuarta, condiciones para el aprendizaje organizacional.

Entenderemos como definición de aprendizaje organizacional la siguiente: Es la capacidad de las organizaciones de crear, organizar y procesar información desde sus fuentes, para producir nuevo conocimiento individual, de equipo, organizacional e inter-organizacional, construyendo una cultura que lo facilite y permitiendo las condiciones para desarrollar nuevas capacidades, diseñar nuevos productos y servicios, incrementar la oferta existente y mejorar procesos, orientados a la sostenibilidad.

Las tipologías de conocimiento determinadas en el modelo son las de mayor relevancia y aplicabilidad al aprendizaje organizacional, y están determinadas por tres tipos de conocimiento: tácito, explícito y virtual. El conocimiento tácito es un saber en acción individual o social, de alta trascendencia en la creación de conocimiento, que determina el "know how"; es difícil de imitar, copiar o medir. El conocimiento explícito es comúnmente tangible. El conocimiento virtual surge en un momento de comprensión compartida, que es provocado por interacciones dirigidas hacia un cierto propósito, tanto individual como colectivamente.

De esta manera en las organizaciones se presenta conversión del conocimiento, el cual es un proceso esencial en el aprendizaje organizacional, que se basa en Nonaka et al. (1999) y Choo (2003). Estos autores plantean que la socialización se da cuando se comparte conocimiento, de tácito a tácito; la exteriorización se da en las organizaciones cuando se comparte conocimiento, de tácito a explícitovirtual; la combinación se presenta en las organizaciones cuando se comparte conocimiento, de explícito a explícito; y finalmente la interiorización se presenta cuando se comparte conocimiento: de explícito a tácito.

En el modelo propuesto por Garzón (2008), ilustrado en la Figura 1, la primera variable se denomina fuentes del aprendizaje organizacional y hace referencia a las distintas situaciones que deben ser consideradas y utilizadas para generar aprendizajes. Estas fuentes de aprendizaje están conformadas por: las crisis y los problemas; los clientes; las unidades especializadas; las adquisiciones; la competencia; la experiencia y los practicantes; la tecnología; las 


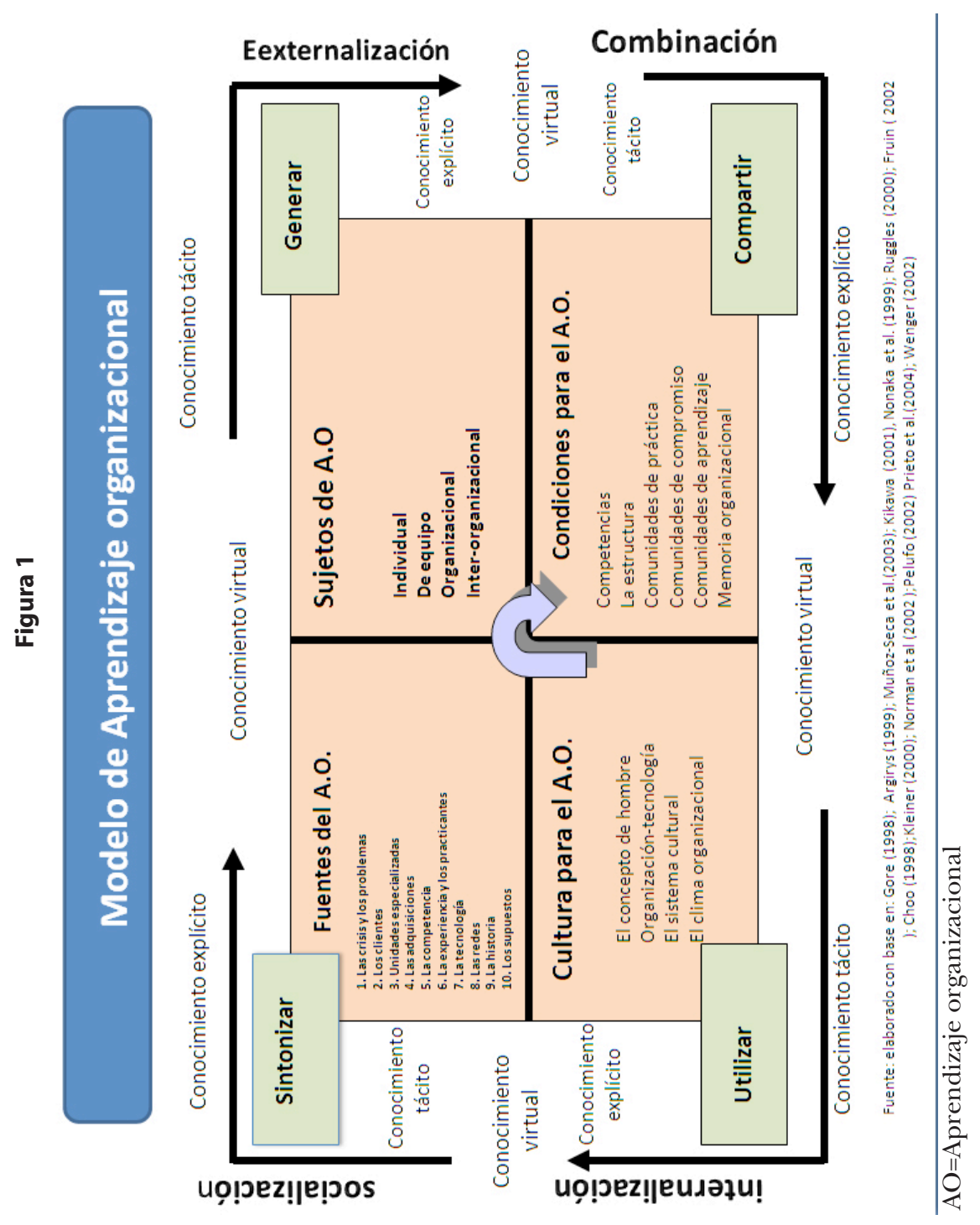


redes; la historia y los supuestos. La segunda variable se denomina sujetos del aprendizaje y estos son: individuos, equipos, organizaciones e inter-organizacional. La tercera variable es la cultura para el aprendizaje organizacional, que se entiende como la conciencia colectiva que se expresa en el sistema de significados compartidos por los miembros de la organización que los identifica y diferencia de otros institucionalizando. Las sub-variables de la cultura organizacional son: el concepto de hombre que tiene el líder de la organización; el sistema cultural y el clima organizacional. La cuarta variable del modelo se denomina condiciones para el aprendizaje organizacional debido a que se hace necesario que el conocimiento sea transmitido y conservado a través de las diferentes condiciones que brinden las organizaciones para la generación del mismo y estas son: competencias; estructura; comunidades de práctica; comunidades de compromiso; comunidades de aprendizaje y memoria organizacional.

\section{Metodología}

Este trabajo empírico se llevó a cabo mediante el análisis de los datos obtenidos a través de un instrumento con escala Likert, aplicado a una muestra de ejecutivos de empresas de Santo Domingo en República Dominicana que estaban en proceso de formación directiva.

La escala tipo Likert utilizada como mecanismo de recolección de la información está desarrollado bajo la metodología pertinente. Esta es un tipo de escala aditiva que corresponde según Padua (1987) a un nivel de medición ordinal, consistente en una serie de ítems o juicios ante los cuales se solicita la reacción del sujeto. El estímulo (ítem o sentencia) representa la propiedad que el investigador está interesado en medir, y las respuestas están solicitadas en el grado de acuerdo o en desacuerdo que el sujeto tenga con la sentencia en particular.

Los pasos seguidos para la construcción de la escala tipo Likert fueron: en primer lugar la elaboración de una lista inicial de aseveraciones relevantes a la variable que se quiere medir; administración de la prueba a una muestra de sujetos que van a actuar como jueces; luego la asignación de puntaje total a los ítems según la dirección 
positiva o negativa de estos (confiabilidad); sometimiento del instrumento a la prueba de validez; análisis de los ítems con base en el cálculo del valor escalar de cada aseveración; eliminación de algunas aseveraciones con el criterio de ambigüedad y de inadecuación; y por último la validación y construcción del instrumento final.

La retroalimentación sobre el cuestionario inicial se realizó con una pre-prueba a través de encuentros personales con altos directivos que permitió confrontar los ítems de medición de las variables de análisis y subsanar posibles errores, ambigüedades y deficiencias de interpretación.

La metodología para el análisis empírico comprendió de dos pasos esenciales: en primer lugar, a fin de validar las fuentes, los niveles de aprendizaje, la cultura y las condiciones para el aprendizaje, se aplicó la técnica de análisis de clusters. El objetivo era segmentar la muestra en función de las cuatro variables del aprendizaje organizacional. En segundo lugar, para analizar el impacto de cada una las variables a saber: las fuentes, los sujetos, la cultura y las condiciones para el aprendizaje, en relación con la capacidad de aprendizaje y su incidencia en los resultados organizativos, se aplicó en cada uno de esos clusters dos análisis uni-variantes (media, desviación típica, valores máximos y mínimo). El primero se aplicó a los determinantes de la capacidad de aprendizaje para validar las fuentes, los sujetos, la cultura y las condiciones para el aprendizaje, y el segundo a los resultados organizacionales, tanto a los económicos como a los no económicos.

Asimismo, se aplicó el análisis de un test ANOVA, que permitió comprobar la significación estadística de las diferencias que se registran entre los valores medios de cada una de las variables y los resultados económicos y resultados no económicos en las cuatro situaciones planteadas.

Estos resultados se completaron con un test de Tukey que permitió identificar las diferencias significativas resultantes de las comparaciones dos a dos entre los cuatro conjuntos utilizando el paquete estadístico SPSS para Windows, Versión 15.0.

La medición de las variables de análisis se llevó a cabo mediante la utilización de escalas múltiples de medición, las cuales incrementaron la confianza acerca de la exactitud y la consistencia de la valo- 
ración. Para la medida de las variables en términos cuantitativos se recurrió principalmente a escalas Likert de cinco posiciones.

La validación del instrumento tipo Likert, requirió la aplicación del análisis factorial de componentes principales con la rotación Varimax, con el paquete estadístico SPSS, y la utilización de escalas múltiples de medición para cada variable, para verificar si los ítems correspondientes se ajustaban al constructo estipulado.

Este análisis de componentes principales permitió resumir la información y la estructura de cada una de las diferentes variables que se necesitan para el análisis. Dicho análisis se realizó individualmente para cada variable mediante pruebas de fiabilidad.

\section{Analisis de los Resultados obtenidos}

La elaboración del instrumento de la investigación se realizó mediante cuadros metodológicos, para posteriormente estructurar la primera versión del instrumento a utilizar en la investigación. En la prueba piloto del instrumento tipo Likert, se escogieron aleatoriamente 35 personas de los directivos que participan en programas de formación directiva en Barna Business School ${ }^{3}$, los cuales conformaron la muestra, considerada representativa según los planteamientos de Isikawa (1985), al plantear que para universos pequeños, una muestra de 35 personas es representativa.

Los resultados obtenidos con el método de componentes principales y el coeficiente de correlación permiten determinar ítems redundantes. Este método los comprime, generando un índice y un coeficiente de correlación y la raíz característica de la varianza de cada ítem para eliminar aquellos redundantes.

Se diseñó un instrumento compuesto de 138 ítems en una escala Likert con los cuales se buscó medir la creación e impulso del factor organizacional. (Ver Apéndice 1). La Tabla 1 muestra la estructura del cuestionario. Un análisis detallado de este ítem permitió determinar dichos factores (Plazas, 2005; citado por Garzón, 2008). Con este primer paso, a partir de una encuesta piloto se evaluó

\footnotetext{
${ }^{3}$ Escuela de Negocios que funciona en República Dominicana en convenio con la Universidad de Barcelona para la doble titulación del MBA.
} 
la pertinencia de los ítems que comprenden el test, buscando ante todo que estos sean determinantes de los factores y por tanto claves en la evaluación de los objetivos. Los ítems se construyeron siguiendo el marco conceptual y la pertinencia de cada ítem en el test, los cuales dependen en gran parte de su formulación y del conocimiento y experiencia del investigador. Algunos ítems de los que presentaron correlación cercana a .90 se excluyeron en el instrumento, por cuanto se creyó que su importancia no era relevante basado en el resultado estadístico.

Para efectos de análisis de los resultados se siguió el siguiente plan de análisis estadístico: una primera parte del análisis se realizó considerando las dimensiones de cada una de las áreas de evaluación relacionadas con el aprendizaje organizacional, posteriormente con base en la matriz de correlación ${ }^{4}$ se descompuso el análisis de componentes principales buscando la generación un nuevo indicador.

Con el objeto de asegurar la contribución del puntaje medio de la dimensión con respecto al total se estableció la correlación entre dichos puntajes. Esta correlación permitió mostrar la pertinencia de la dimensión, como variable de análisis en el contexto del aprendizaje organizacional, y la obtención de los siguientes resultados (ver Tabla 2):

Variable cultura para el aprendizaje organizacional: para esta variable se diseñaron 26 afirmaciones (ver cuadro metodológico), y con la aplicación de los datos estadísticos, componentes principales y coeficiente de relación como se puede ver la matriz de coeficiente de correlación de cultura para el aprendizaje organizacional en la Tabla 3, se logró eliminar las preguntas redundantes, y quedaron siete (7) afirmaciones con un índice del $24.53 \%$, confirmada en la Tabla 4 con el resultado de la aglomeración de los clusters y cuyos resultados se pueden ver en la Figura 2, en el dendograma del coeficiente de correlación de cultura para el aprendizaje organizacional.

${ }^{4}$ Sirve para determinar cuáles items contribuyen más a explicar el concepto. 


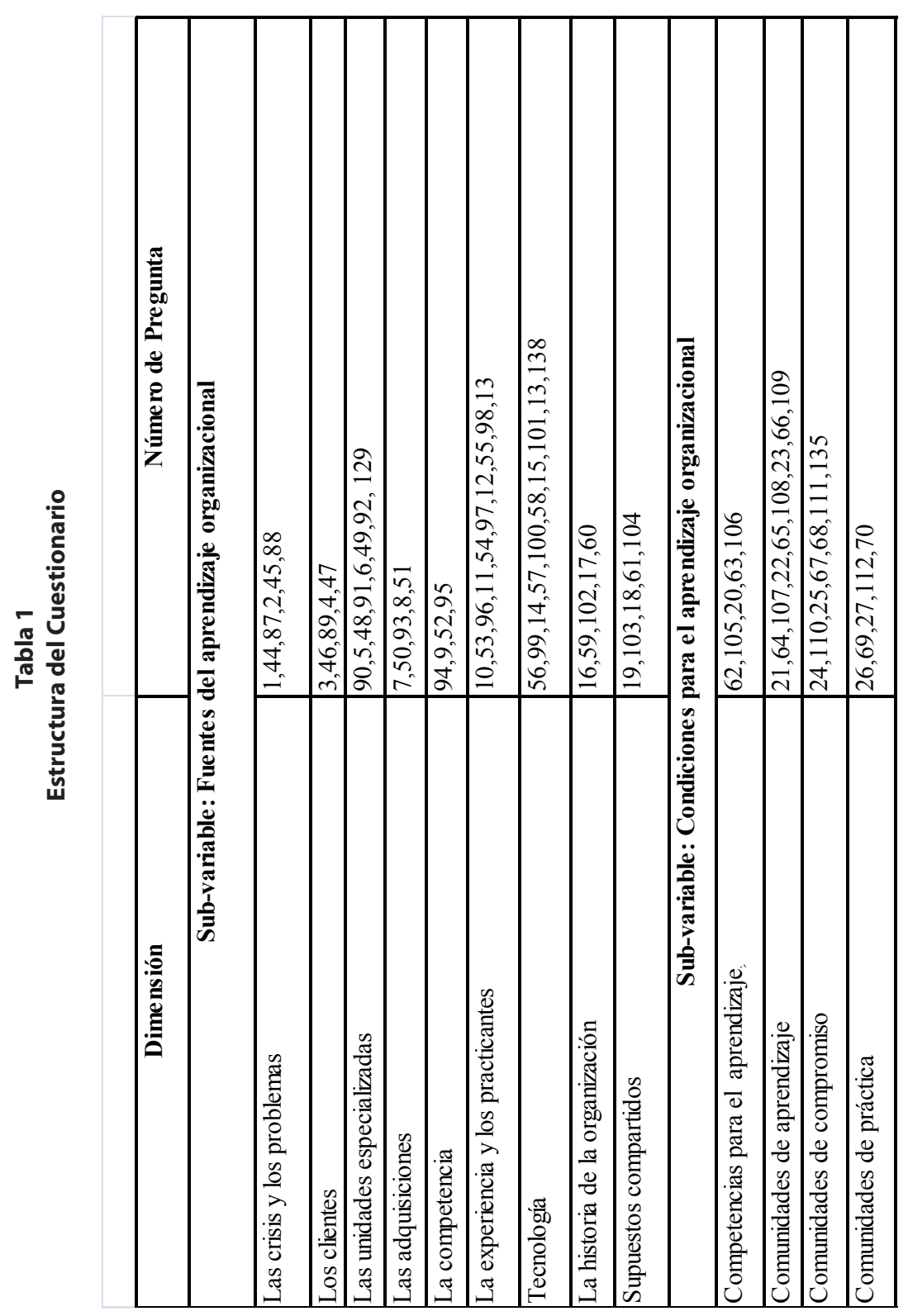




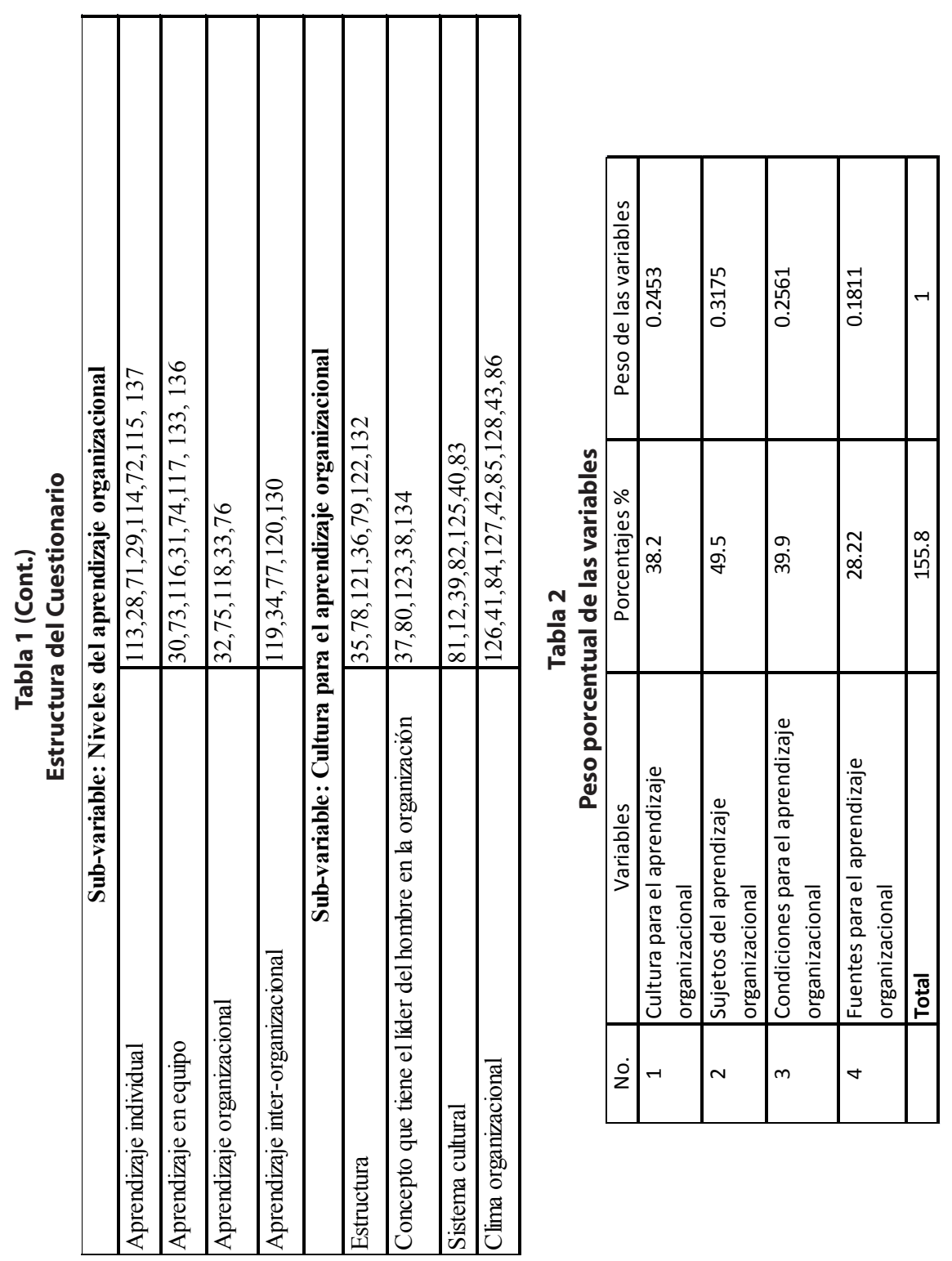




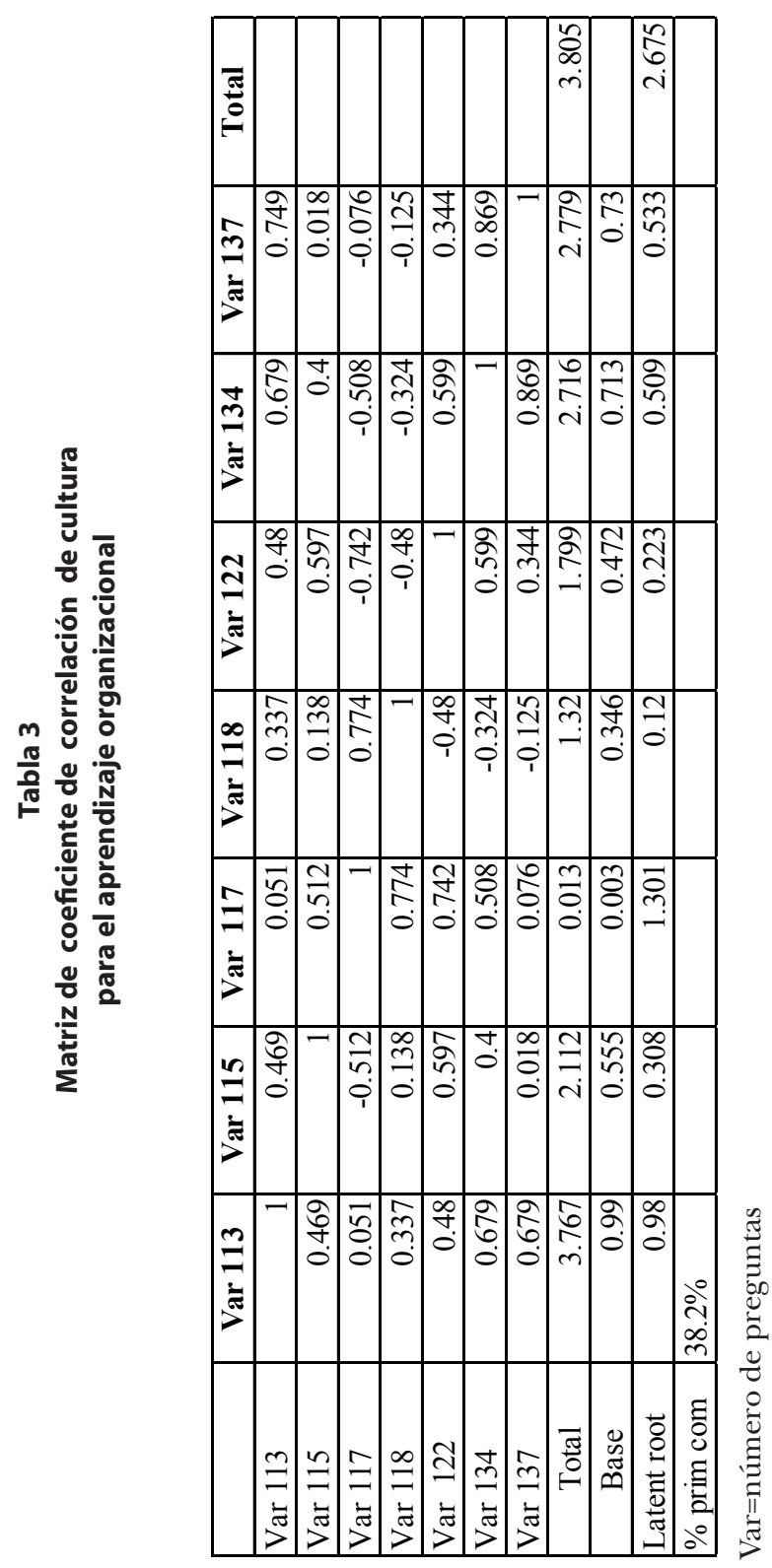


Tabla 4

Aglomeración de los clusters

\begin{tabular}{|c|c|c|c|}
\hline \multirow{2}{*}{ Etapa } & \multicolumn{2}{|c|}{ Combinación de cluster } & \multirow{2}{*}{$\begin{array}{c}\text { Coeficiente de } \\
\text { correlación }\end{array}$} \\
\cline { 2 - 3 } & Cluster uno & Cluster dos & 0.8690 \\
\hline 1 & 134 & 137 & 0.7740 \\
\hline 2 & 117 & 118 & 0.7490 \\
\hline 3 & 113 & 137 & -0.7420 \\
\hline 4 & 117 & 122 & 0.6790 \\
\hline 5 & 113 & 134 & 0.5990 \\
\hline 6 & 122 & 134 & 0.5970 \\
\hline 7 & 115 & 122 & \\
\hline
\end{tabular}

Figura 2

Dendograma del coeficiente de correlación de cultura para el aprendizaje organizacional

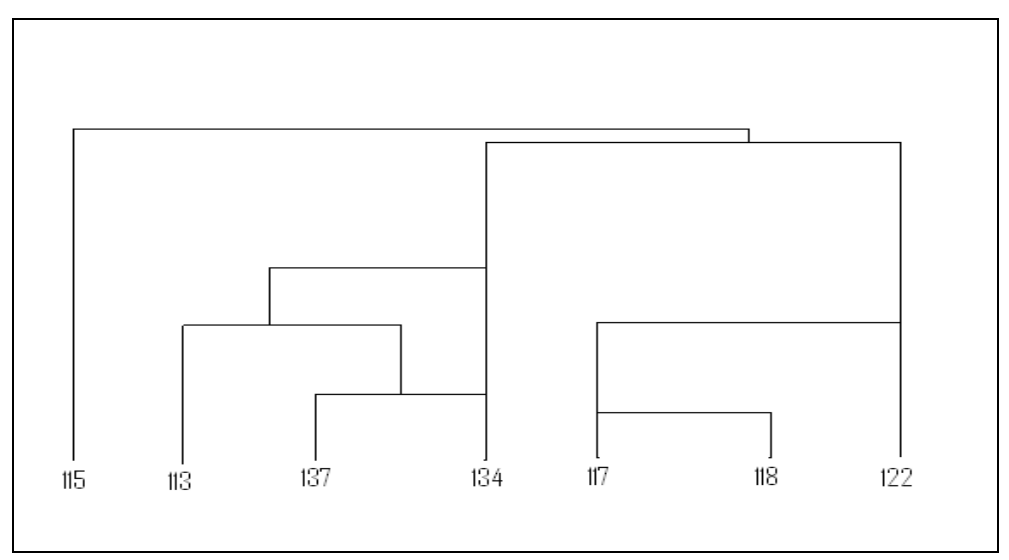

Para la variable sujetos para el aprendizaje organizacional: se diseñaron 22 afirmaciones, y con la aplicación de los datos estadísticos, componentes principales y coeficiente de relación, se logró eliminar las preguntas redundantes, y quedando cinco (5) afirmaciones con un índice del $31.7 \%$, confirmada en la Tabla 5 matriz de coeficiente de correlación de sujetos del aprendizaje organizacional con el resultado de aglomeración de los clusters de la Tabla 6 y cuyos resultados se pueden ver en la Figura 3, en el dendograma del coeficiente de correlación de sujetos del aprendizaje organizacional. 


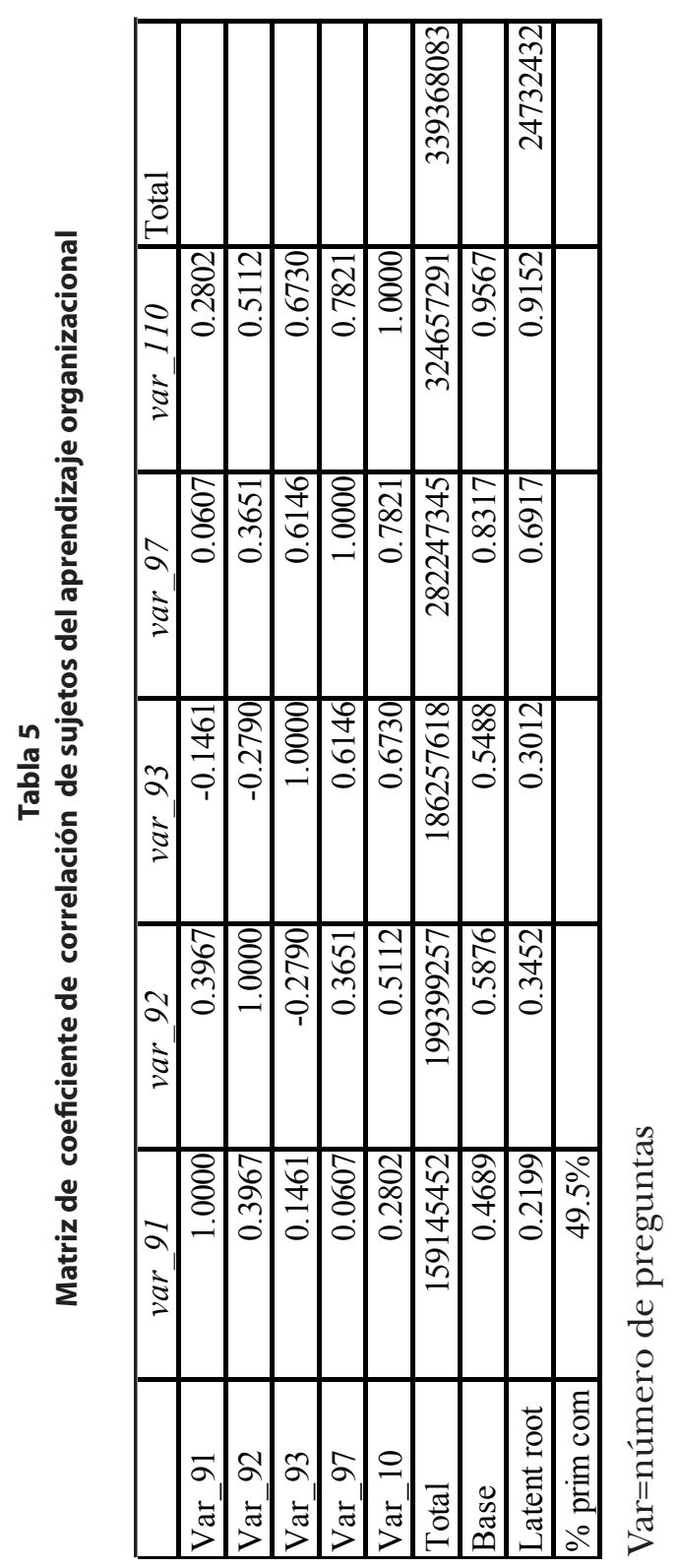


Tabla 6

Aglomeración de los clusters

\begin{tabular}{|c|c|c|c|}
\hline \multirow{2}{*}{ Etapa } & \multicolumn{2}{|c|}{ Combinación de cluster } & \multirow{2}{*}{$\begin{array}{c}\text { Coeficiente de } \\
\text { correlación }\end{array}$} \\
\cline { 2 - 3 } & Cluster uno & Cluster dos & 0.7821 \\
\hline 1 & 97 & 110 & 0.6730 \\
\hline 2 & 93 & 110 & 0.6140 \\
\hline 3 & 93 & 97 & 0.5110 \\
\hline 4 & 92 & 110 & 0.3960 \\
\hline 5 & 91 & 92 & \\
\hline
\end{tabular}

Figura 3

Dendograma del coeficiente de correlación de sujetos del aprendizaje organizacional

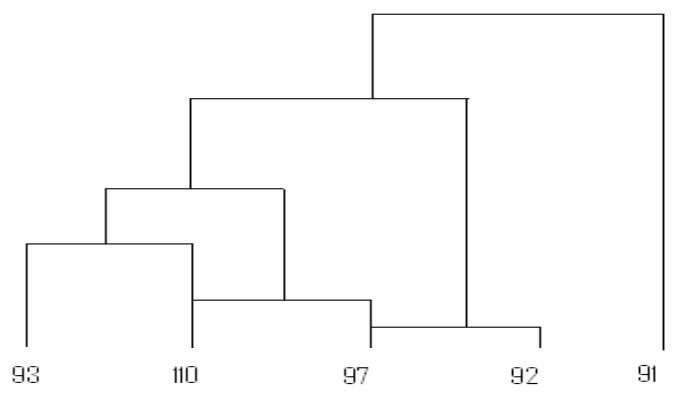

Para la variable condiciones para el aprendizaje organizacional: se diseñaron 25 afirmaciones (ver cuadro metodológico), y con la aplicación de los datos estadísticos, componentes principales y coeficiente de relación, se logró eliminar las preguntas redundantes y quedaron seis (6) afirmaciones con un índice del 25.6\%, confirmada en la Tabla 7 matriz de coeficiente de correlación de sujetos del aprendizaje organizacional con el resultado de aglomeración de los clusters de la Tabla 8 y cuyos resultados se pueden ver en la Figura 4, en el dendograma del coeficiente de correlación de condiciones para el aprendizaje organizacional. 


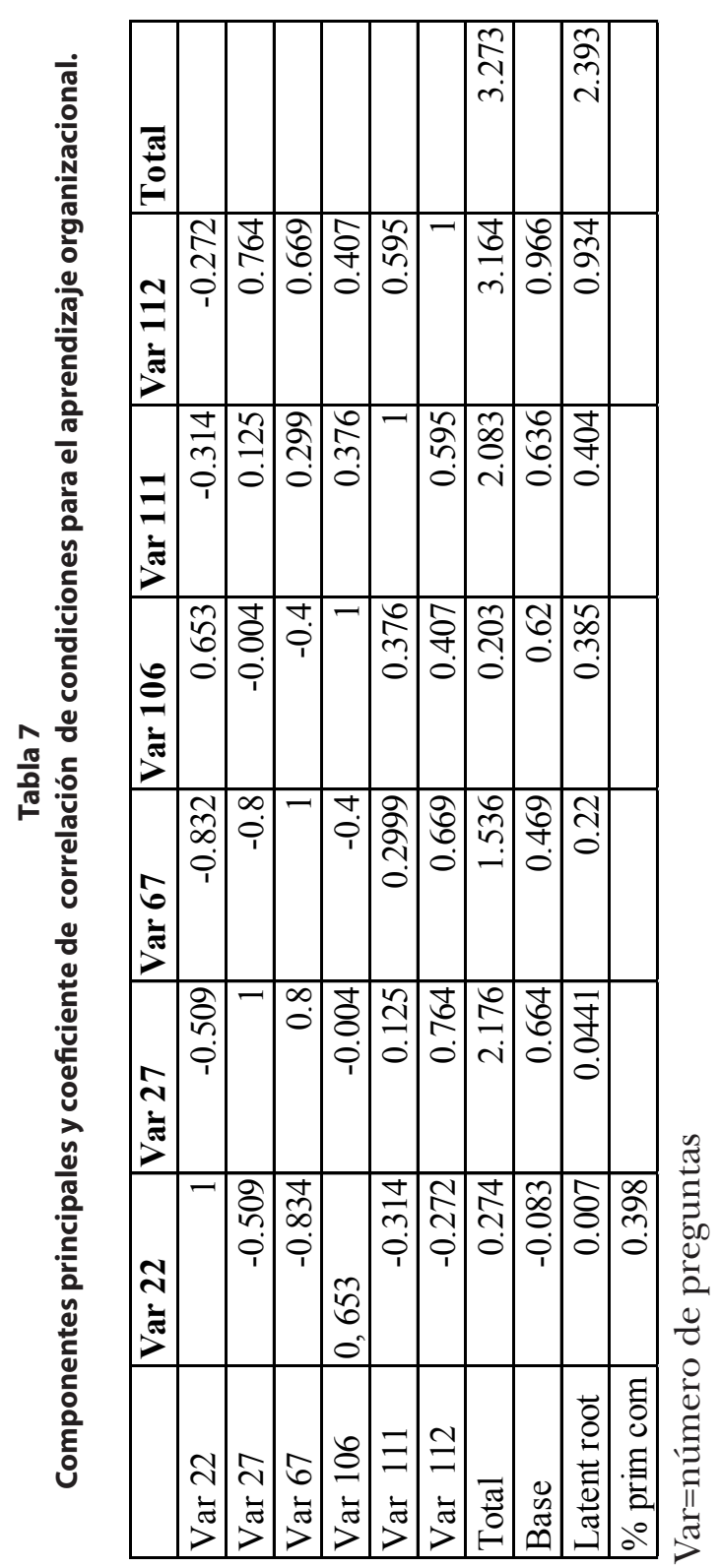


Tabla 8

Aglomeración de los clusters

\begin{tabular}{|c|c|c|c|}
\hline \multirow{2}{*}{ Etapa } & \multicolumn{2}{|c|}{ Combinación de cluster } & \multirow{2}{*}{$\begin{array}{c}\text { Coeficiente de } \\
\text { correlación }\end{array}$} \\
\cline { 2 - 3 } & Cluster uno & Cluster dos & -0.832 \\
\hline 1 & 22 & 67 & 0.800 \\
\hline 2 & 27 & 67 & 0.764 \\
\hline 3 & 27 & 112 & 0.669 \\
\hline 4 & 67 & 112 & 0.653 \\
\hline 5 & 22 & 108 & 0.590 \\
\hline 6 & 111 & 112 & \multicolumn{2}{c}{} \\
\hline
\end{tabular}

Figura 4

Dendograma del coeficiente de correlación de sujetos

para el aprendizaje organizacional

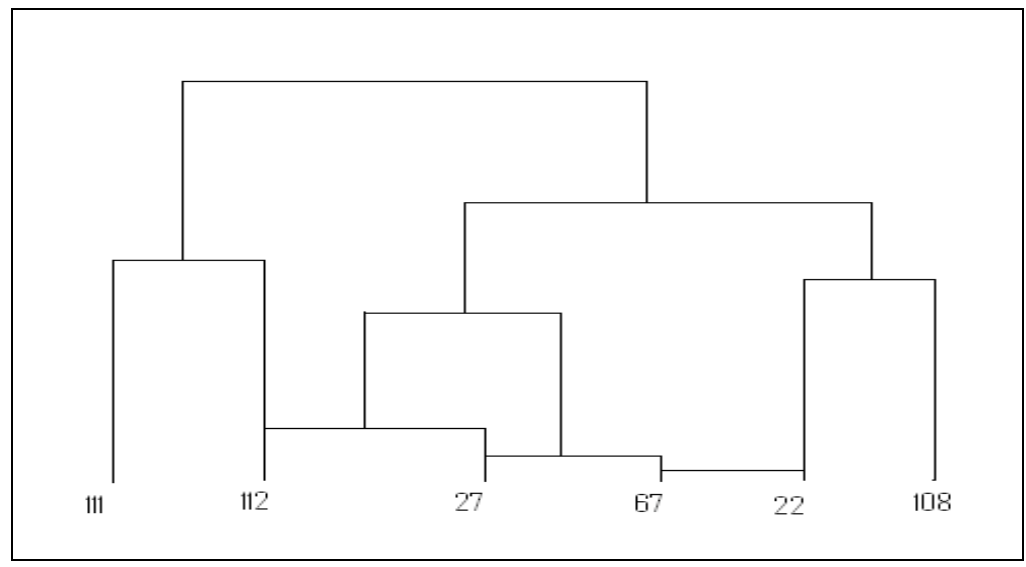

Para la variable fuentes para el aprendizaje organizacional: se diseñaron cincuenta y seis (56) afirmaciones (ver cuadro metodológico), y con la aplicación de los datos estadísticos, componentes principales y coeficiente de relación, se logró eliminar las preguntas redundantes, y quedaron diez y nueve (19) afirmaciones con un índice del $18.11 \%$, confirmada en la Tabla 9 matriz de coeficiente de correlación de sujetos del aprendizaje organizacional con el resultado de aglomeración de los clusters de la Tabla 10 y cuyos resultados 
se pueden ver en la Figura 5, en el dendograma del coeficiente de correlación de sujetos para el aprendizaje organizacional.

El rediseño del instrumento se realizó basado en los resultados anteriores, elaborando nuevos cuadros metodológicos y la nueva versión del instrumento propuesto para la investigación (ver Apéndice 1). Del análisis realizado surgió el rediseño del instrumento en tres variables que agrupan a las fuentes la cultura y las condiciones para el aprendizaje organizacional. Las cargas o correlaciones establecidas para cada una de las variables con los tres componentes del sistema original permitió ratificar que las variables están más identificadas con el factor uno que con los demás factores. Por esta razón, con el fin de ver la afinidad de las variables con los tres factores simultáneamente, se rotan los ejes de sistema factorial de forma ortogonal.

Adicionalmente, se encontró que las variables que no tienen representación en ninguno de los tres factores son las que corresponden a los sujetos del aprendizaje organizacional, por tanto no se incluyen en el instrumento final a utilizar en esta investigación.

Con la matriz de factores y preguntas que se muestra en la Tabla 9, se identificó la afinidad que las variables tienen con cada factor y cuáles pueden ser suprimidas del instrumento de medición. Para ellos se seleccionan las variables que tienen correlaciones mayores a 0.6 con cualquiera de las 3 componentes.

De las 138 preguntas se seleccionaron las que mostraron una carga mayor a 0.6 y comunidades de comportamiento superiores al 50.0 $\%$ entre las demás variables. Gracias a esta identificación empírica se observa que los 3 factores son como lo muestra la Tabla 10: Cultura para el aprendizaje organizacional, Fuentes para el aprendizaje organizacional y Condiciones para el aprendizaje organizacional.

El factor Cultura para el aprendizaje organizacional está explicado por las respuestas del indicador de Cultura para el aprendizaje organizacional y con las preguntas numeradas en el nuevo instrumento como 11,13,15,17,19,30.

El factor Fuentes para el aprendizaje organizacional está explicado por las respuestas del indicador de fuentes del aprendizaje organizacional y con las preguntas numeradas en el nuevo instrumento como $6,8,10,12,14,16,18,20,21,22,23,24,25,26,35,36,37,38,39,40$. 
El factor Condiciones para el aprendizaje organizacional está explicado por las respuestas del indicador de condiciones para el aprendizaje organizacional y con las preguntas numeradas en el nuevo instrumento como 1,2,3,4,5,7,9,25,27,28,29,31,33,34.

Todas las preguntas relacionadas con los sujetos del aprendizaje organizacional fueron eliminadas en el nuevo instrumento porque mostraron una carga mayor a 0.6. Hasta aquí se determina la forma de simplificar las 138 variables en tres factores. 


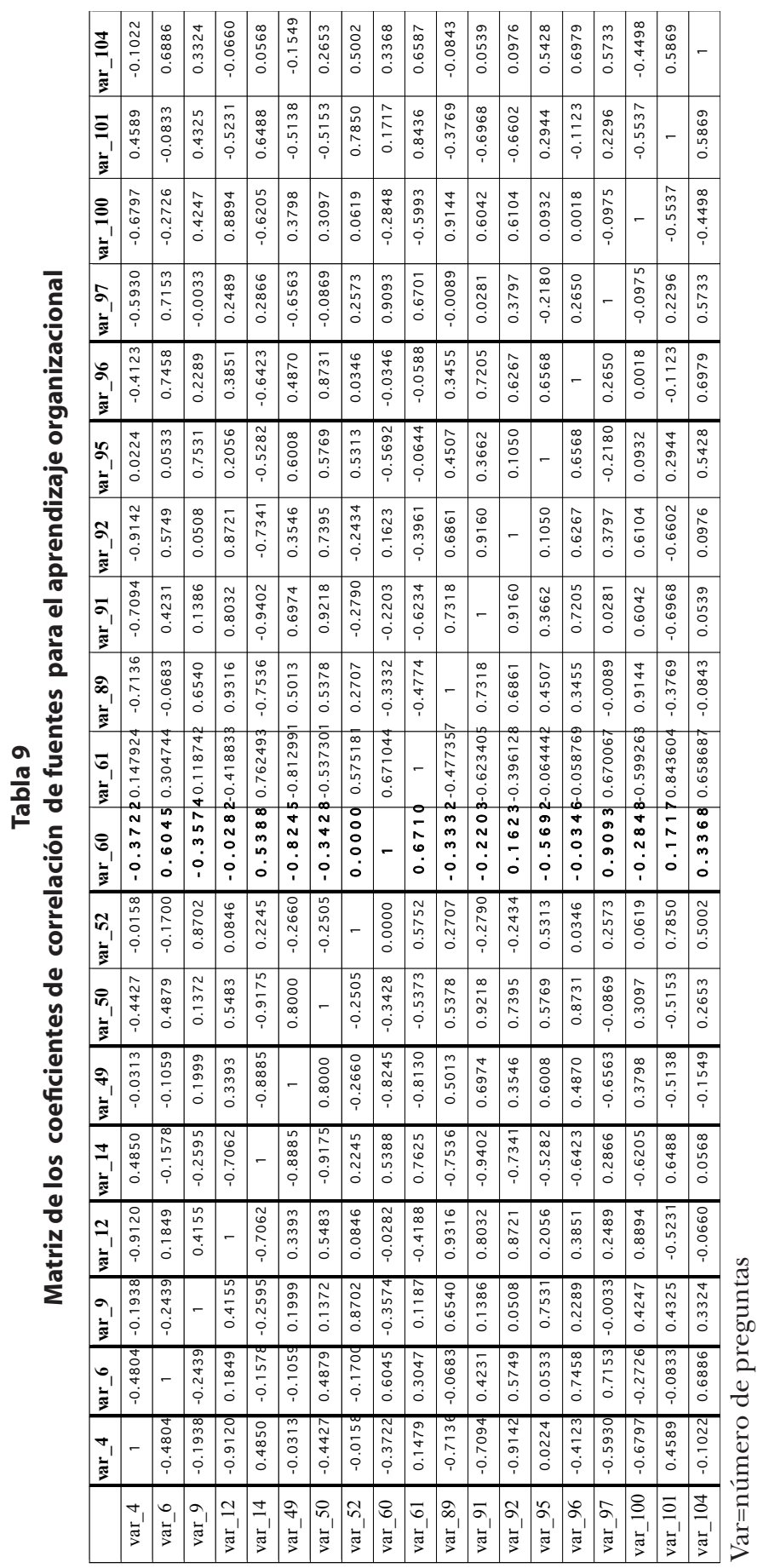




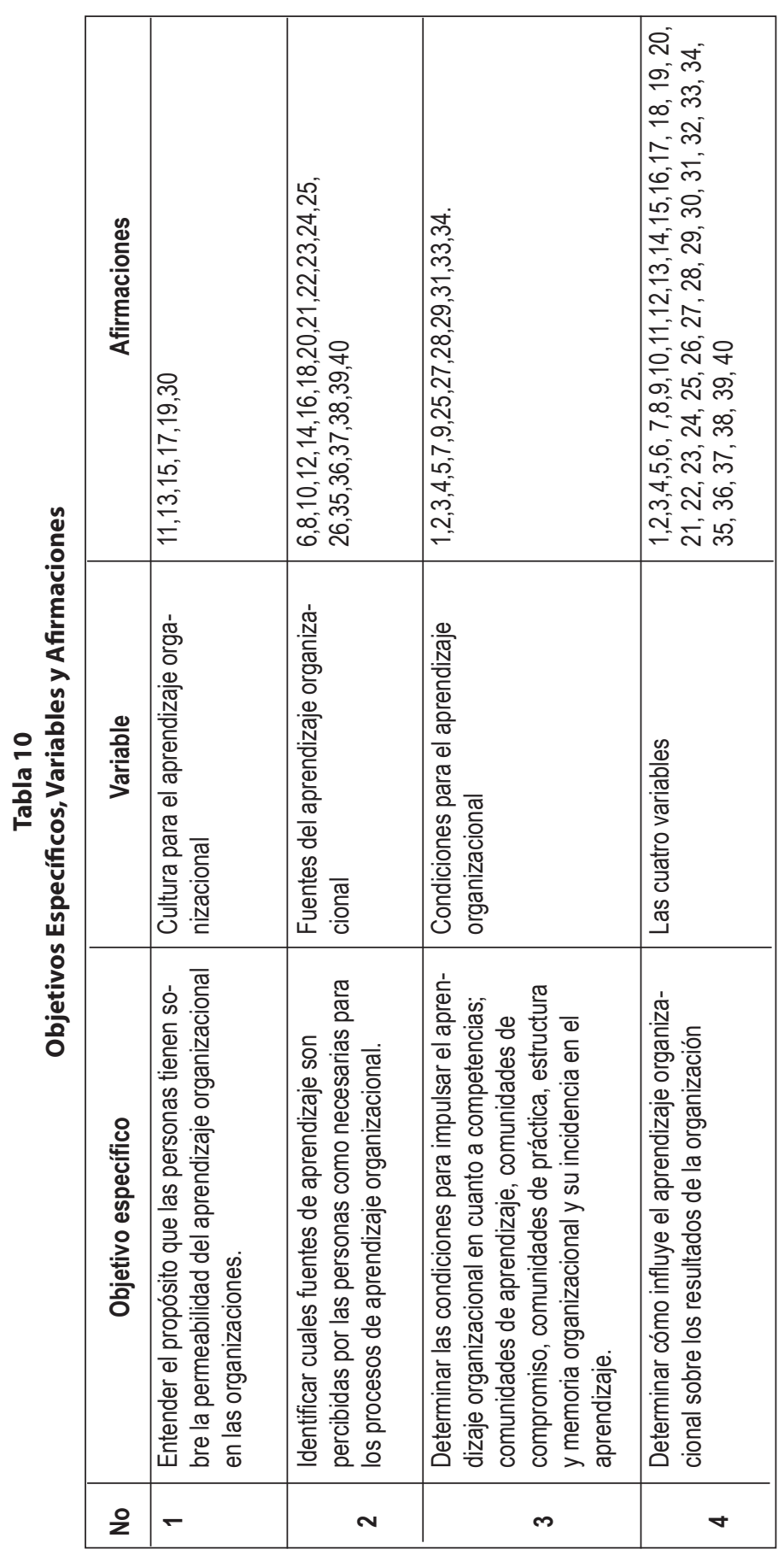




\section{Conclusiones}

Una primera aproximación al aprendizaje organizacional confirma la necesidad que tienen hoy las empresas, y en general las organizaciones, de nuevas herramientas de gestión que permitan abordar nuevas realidades a las que se enfrentan las personas en su día a día y especialmente con la forma de crear valor. El aprendizaje organizacional es pertinente porque el conocimiento es la capacidad para crear lazos más estrechos con los clientes, la capacidad para analizar informaciones corporativas y atribuirles nuevos usos, la capacidad para crear procesos que habiliten a los trabajadores de cualquier lugar a acceder y utilizar información para conquistar nuevos mercados y, finalmente, la capacidad para desarrollar y distribuir productos y servicios para estos nuevos mercados de forma más rápida y eficiente que los competidores.

El objetivo general planteado para este estudio, que fue diseñar instrumentos que permitieran caracterizar la capacidad de aprendizaje organizacional y determinar cómo influyen sobre los resultados de las organizaciones, ${ }^{5}$ se logró al poder identificar la afinidad de las variables con cada factor entre las que se incluyeron las que tienen correlaciones mayores a .6 con cualquiera de los tres componentes.

Las cargas o correlaciones establecidas para cada una de las variables con los tres componentes del sistema original permite ratificar que las variables están más identificada con el factor uno que con los demás factores. Por esta razón, con el fin de ver la afinidad de las variables con los tr es factores simultáneamente se rotan los ejes de sistema factorial de forma ortogonal, de lo cual se encontró que las variables que no tienen representación en ninguno de los tres factores son las que corresponden a los sujetos del aprendizaje organizacional, por tanto no se incluyen en el instrumento final a utilizar en la investigación.

La aplicación de las pruebas estadísticas ha permitido determinar la forma de simplificar las 138 variables en tres factores a saber: la cultu-

\footnotetext{
${ }^{5}$ En este artículo se presentan los resultados de la prueba piloto de un instrumento que se utilizó en un estudio realizado en Brasil, República Dominicana y Colombia (2009).
} 
ra para el aprendizaje organizacional, las fuentes para el aprendizaje organizacional y las condiciones para el aprendizaje organizacional.

Finalmente el rediseño del instrumento se realizó elaborando nuevos cuadros metodológicos y la nueva versión del instrumento propuesto para la investigación. Del análisis anterior se logró el rediseño de un instrumento confiable en tres variables que agrupan a las fuentes para el aprendizaje organizacional y las condiciones para el aprendizaje organizacional, que permitirán caracterizar la capacidad del aprendizaje organizacional. 


\section{RefERENCIAS}

Argyris, C. \& Schön S. (1978). Organizational learning: A theory in action perspective. Reading, M.A.: Addison-Wesley.

Argyris, C. (1999). Sobre el aprendizaje organizacional. México D.F.: Editorial Oxford Press.

Brontis, N. (1999). La gestión humana y el aprendizaje organizacional. Intellectual Capital. MC Master University, Canadá.

Cyert, R. \& March, J. (1963). A behavioral theory of the firm. Englewood Cliffs, N.J.: Prentice-Hall.

Choo Chu, W. (1998). La organización inteligente. México D.F.: Editorial Oxford Press.

Dogson, M. (1993). Organization learning, review of some literatures. Organization Studies, 14(3), 375-394.

Fiol, M. \& Liles, M. (1985). Organizational learning. Academy of Management Review, 10(4), 803-013.

Follett, M. P. (1960). Dynamic administration: The collected papers of Mary Parker Follett. E. M. Fox \& L. Urwick, Eds. New York: Pitman.

Fruin, M. (1996). Knowledge works. New York: Oxford Press.

Garzón, M. A. (2008). Marco teórico del aprendizaje organizacional. Avance de tesis posdoctoral. Presentado en la Universidad de Sao Paulo, Brasil.

Gore, E. (1998). La educación en la empresa: Aprendiendo en contextos organizativos. España: Editorial Granica.

Hall, R. (1986). Organización estructura y procesos. México: Prentice Hall.

Harung, H. S. \& Harung, L. M. (1995). Enhancing organizational performance by strengthening diversity and unity. Learning Organization Journal, 2(2), 9-19.

Hornby, D. \& Thomas, R. (1989). Towards a better standard of management? Personnel Management, 21(1), 48-61.

Kleiner, A. (2000). El cambio basado en el aprendizaje. Realidades sobre la transformación. México D.F., Oxford Press.

Koffman, F. \& Senge, P. M. (1993). Communities of commitment: The heart of learning organizations. Organizational Dynamics, 22(2), 5-23. 
March, J. G. (1991). Exploration and exploitation in organizational learning. Organization Science, 2, 71.

Muñoz-Seca, B. et al. (2003). Del buen hacer y el buen pensar. Madrid: Editorial McGraw Hill.

Nonaka, I. \& Takeuchi, H. (1999). La organización creadora de conocimiento. Cómo las compañias japonesas crean la dinámica de la innovación. (M. Hernández Kocka, Trad.). México: Oxford University Press.

Norman, O. et al. (2002). Knowledge management best practice in national sport organizations. International Journal of Sport Management and Marketing, 2(3), 264-280.

Palacios, M. (2000). Processes and strategies. Revista Hitos de Ciencias Económico Administrativas, 6(15), 31-39.

Padua, J. (1987). Técnicas de investigación aplicadas a las ciencias sociales. México D.F.: Fondo de Cultura Económica.

Peluffo, M. et al. (2002). Introducción a la gestión del conocimiento y su aplicación al sector público. Santiago de Chile: Editorial ILPES.

Prieto, I. et al. (2004). La naturaleza dual de la gestión del conocimiento: implicaciones para la capacidad de aprendizaje y sus resultados organizativos. Revista Latinoamericana de Administración, 32, 47-75.

Revans, R. W. (1984). The origins and growth of action learning. London: Chartwell-Bratt.

Ruggles, Rudy et al. (1999). La ventaja del conocimiento. México: Editorial CECSA.

Vera, A. et al. (2000). Situated action: A symbolic interpretation. Cognitive Science, 17(1) 7-48.

Wagner, E. (2002). The new frontier of learning object design. The Learning Development Journal. Disponible en: www.elearningguild.com.

Zenick, N. (1999). "Ten years' hard study in return of honor" is the belief rooted in Chinese society. ...Learning. Sloan Management Review, 38(1), 9-20. 


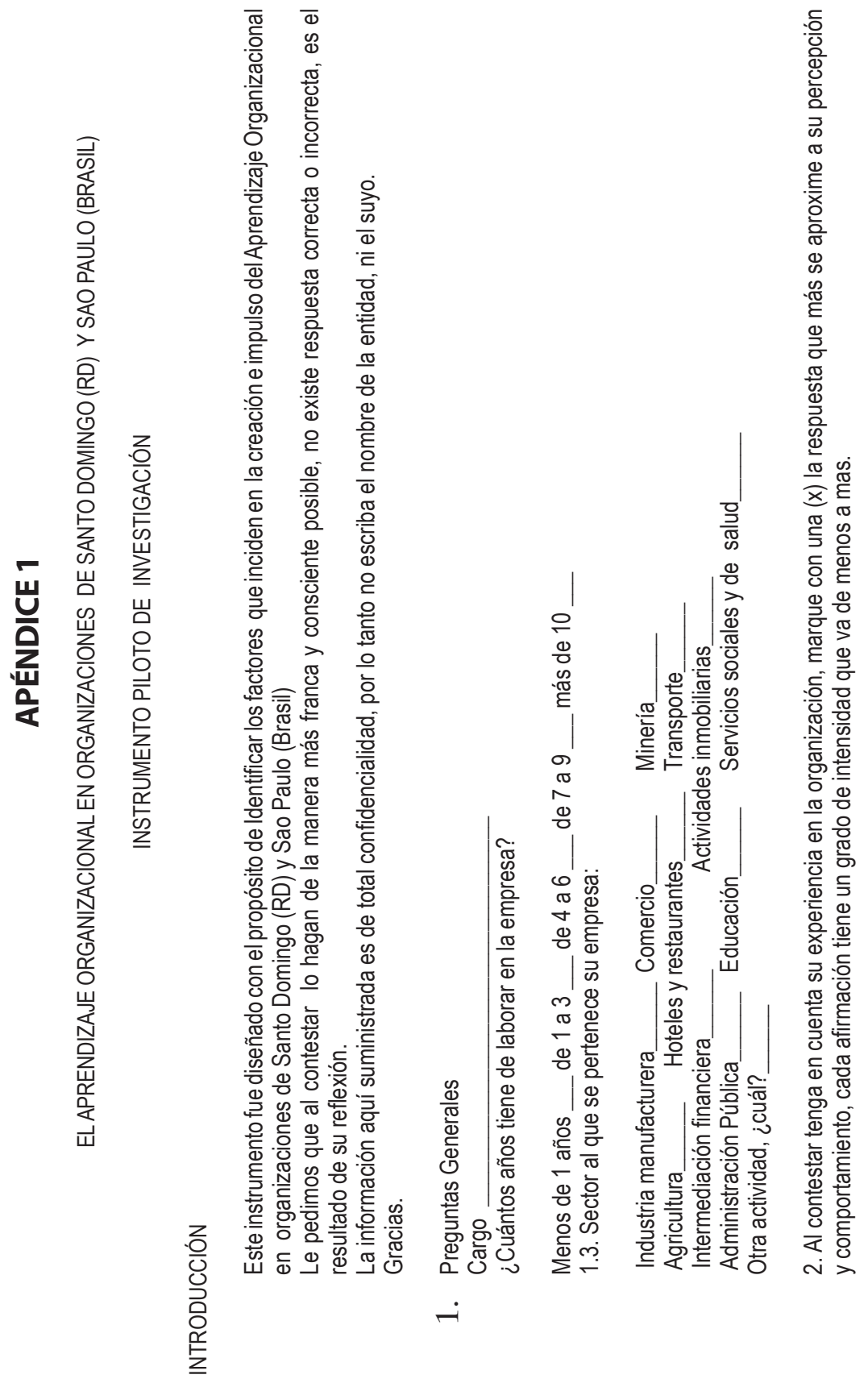


Garzón CAstrillon / Fischer
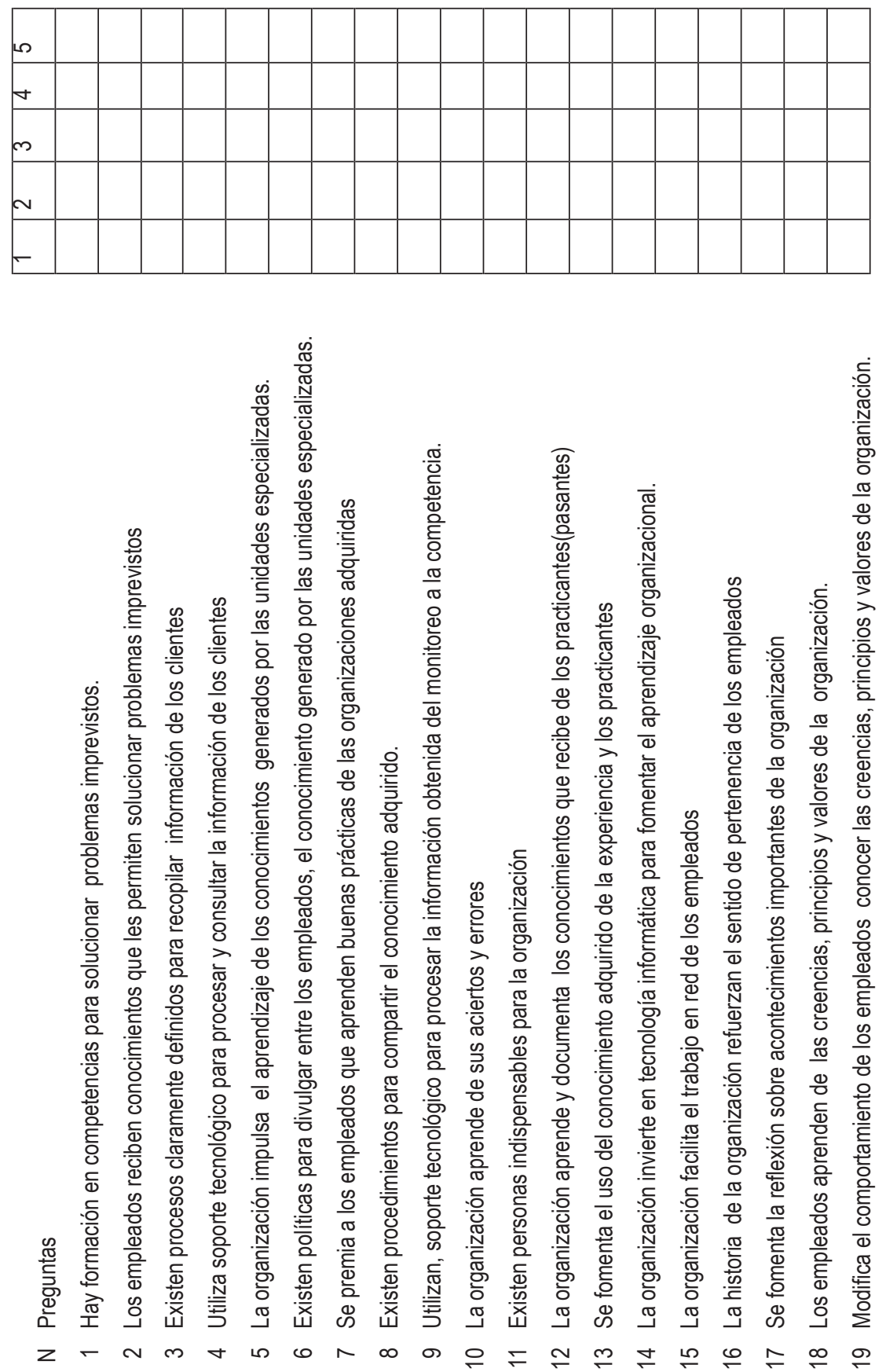

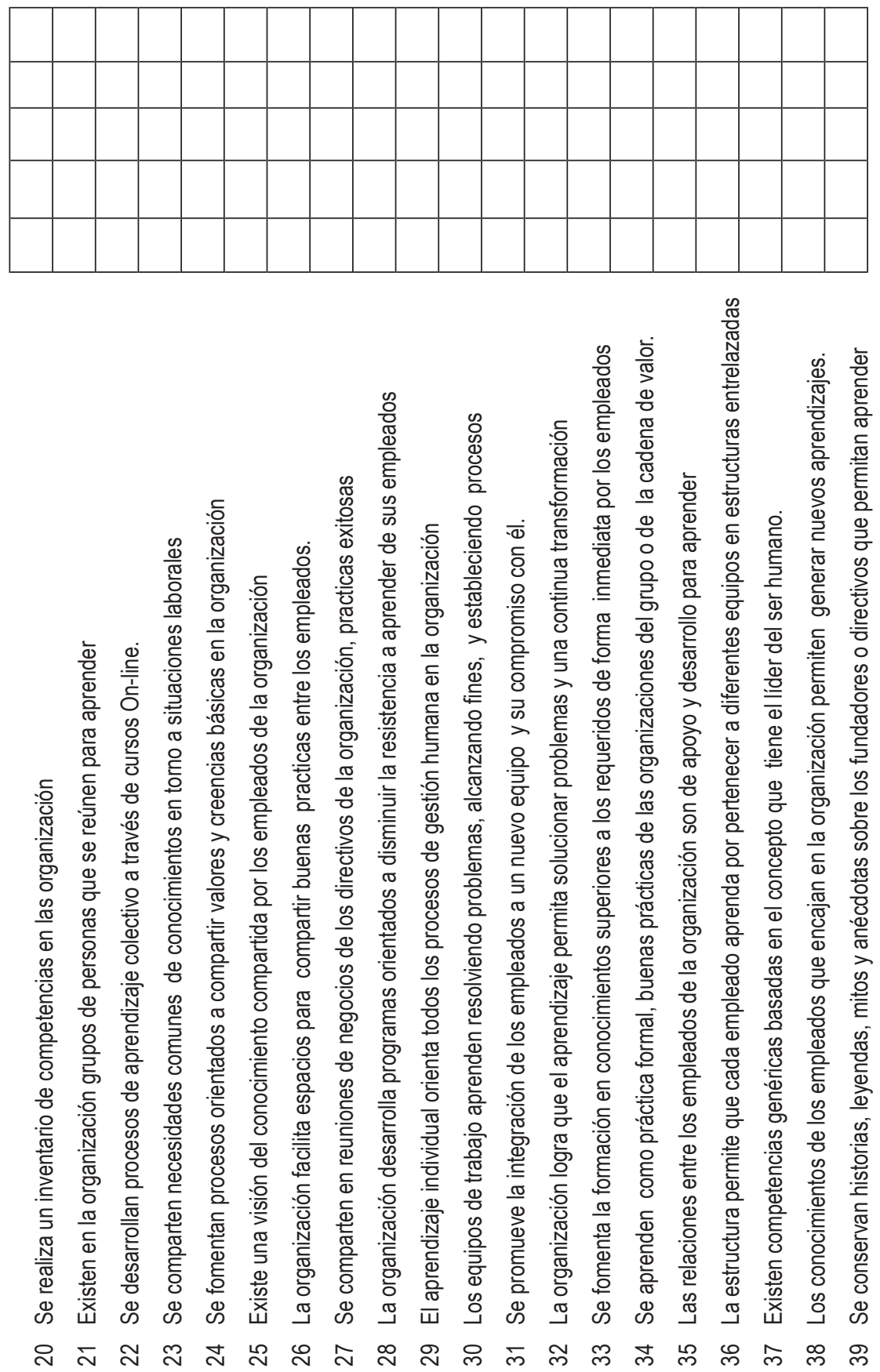

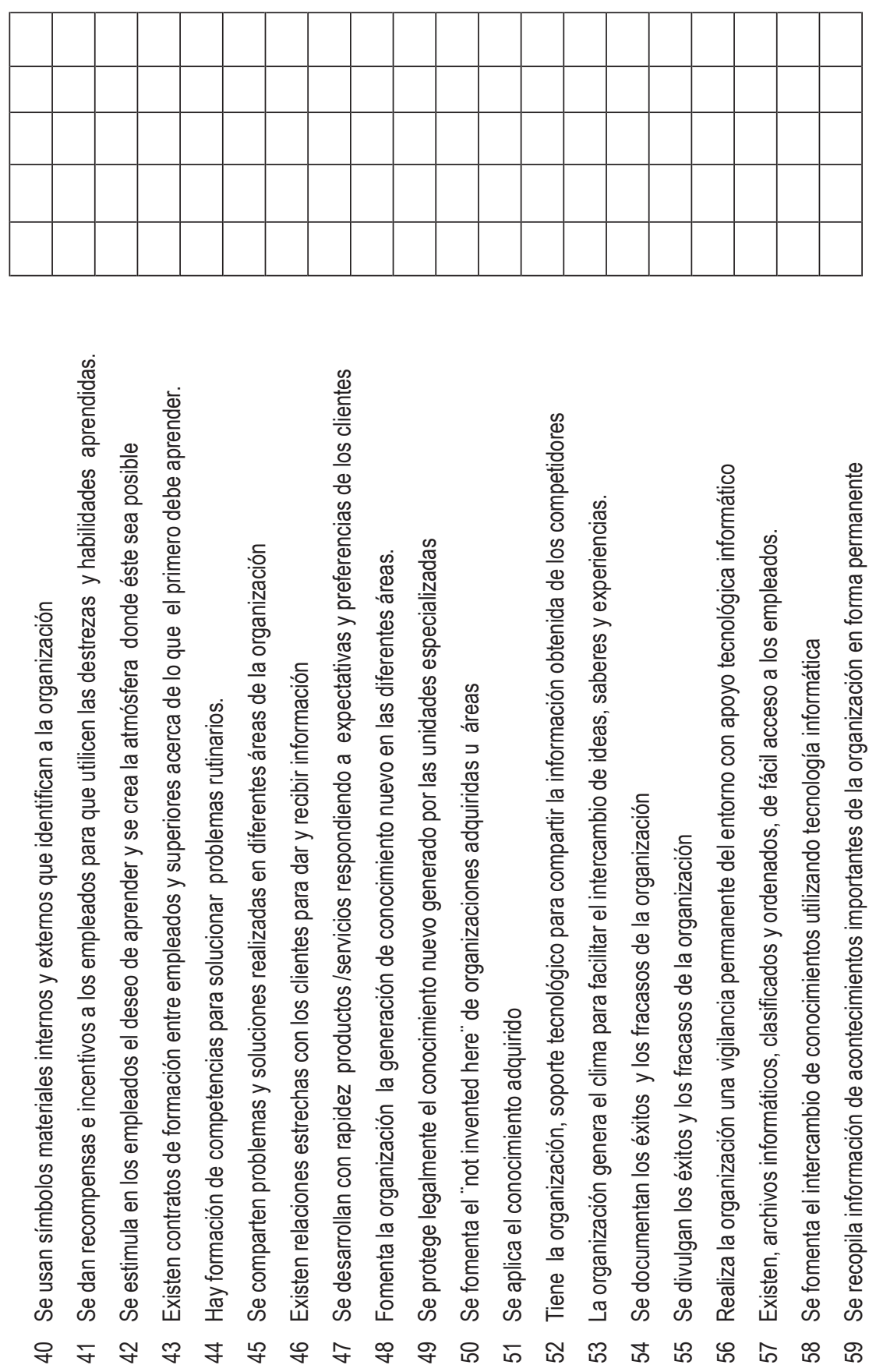

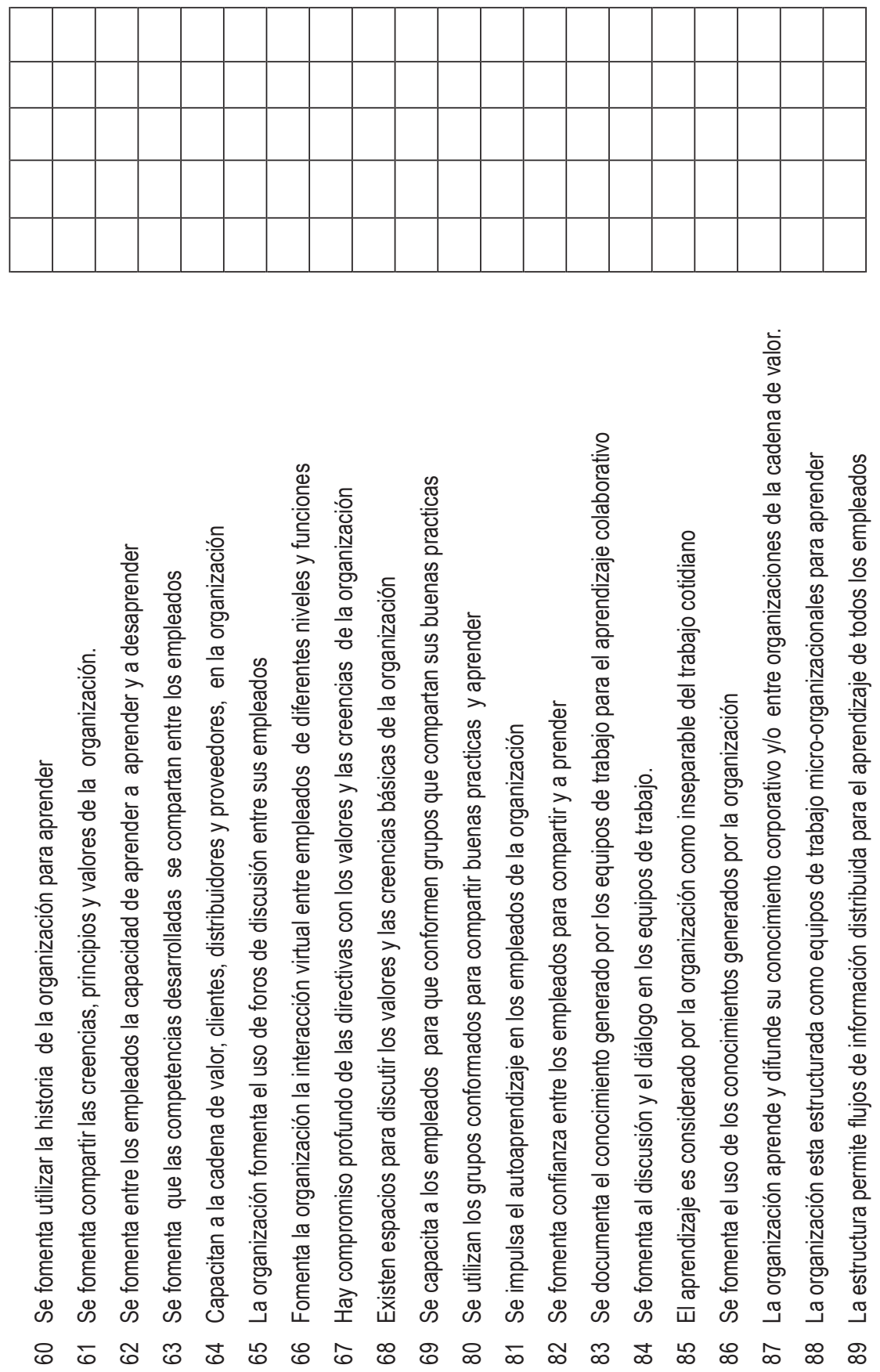

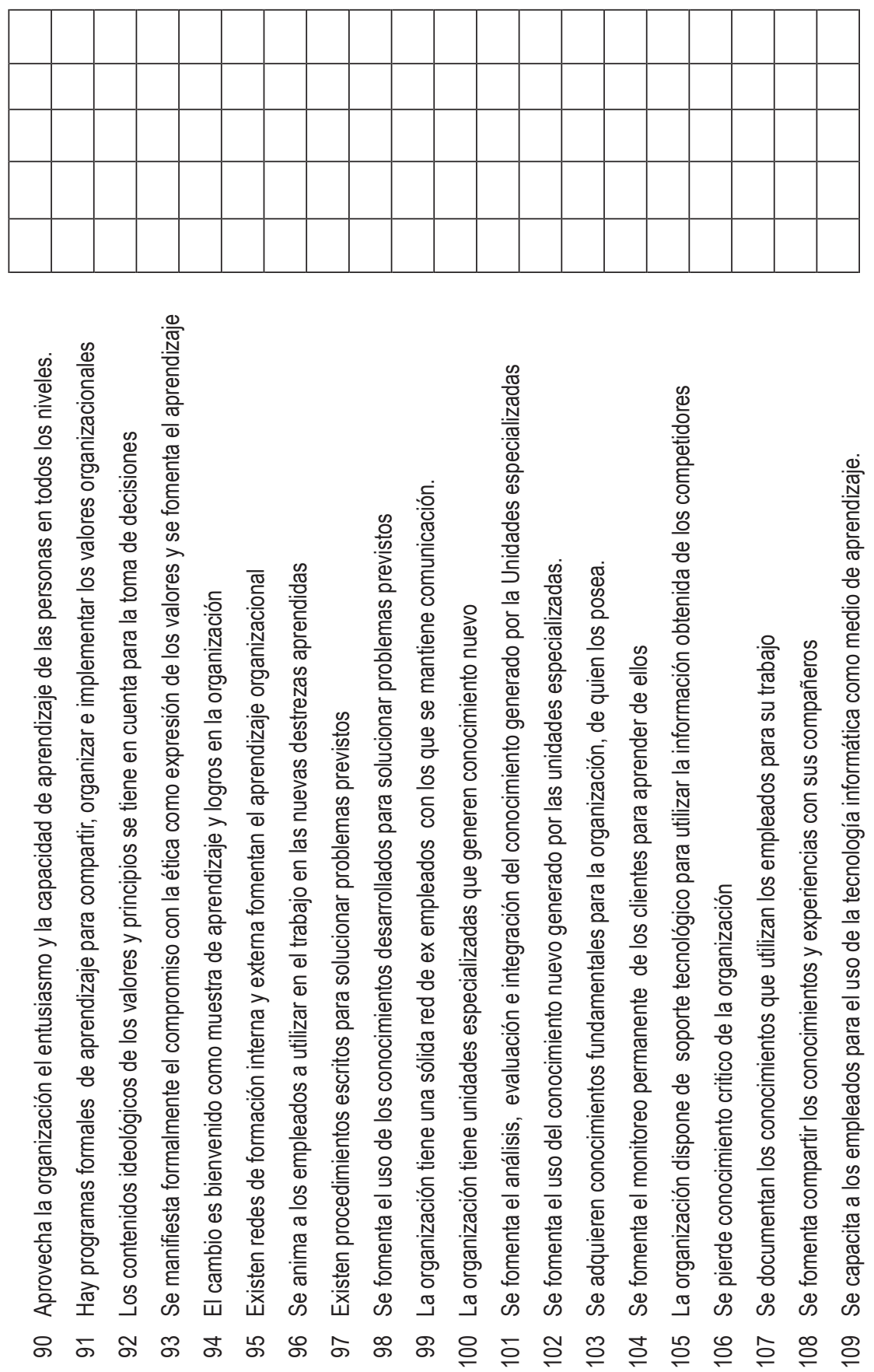


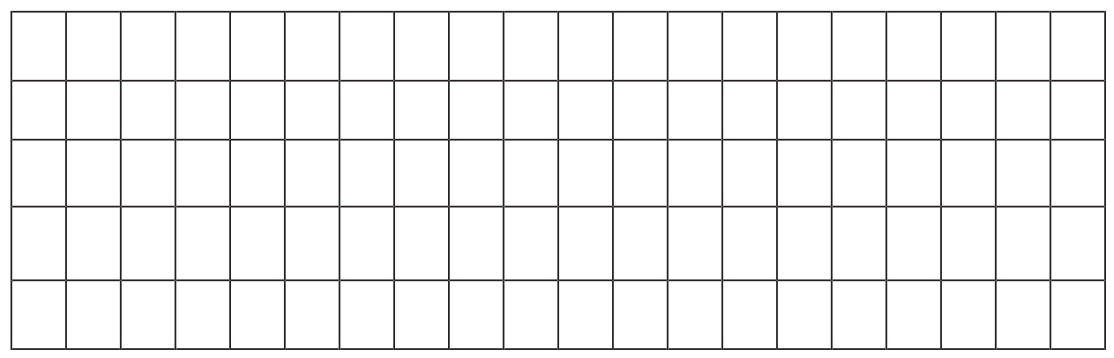

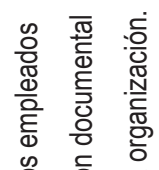

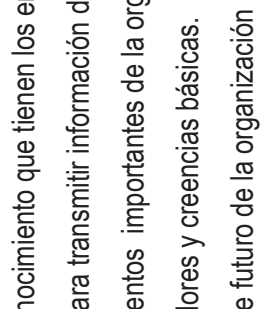

రั

ब

등 응 응

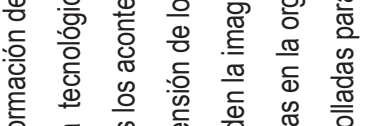

을 敢

ठ ठ

: $\frac{\pi}{0}$ 范

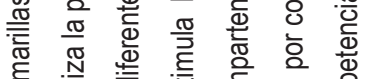

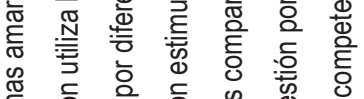

苋

苂

ธ

芭

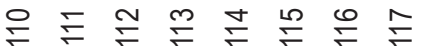

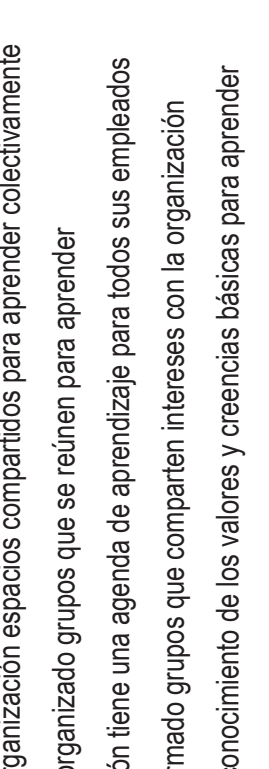

하을

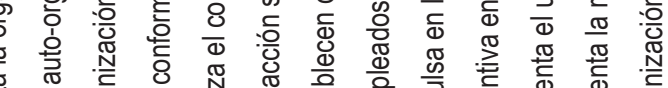

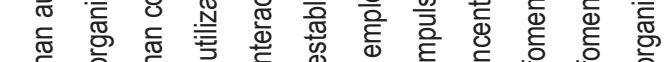

œ

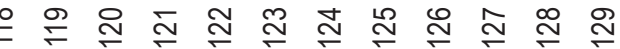



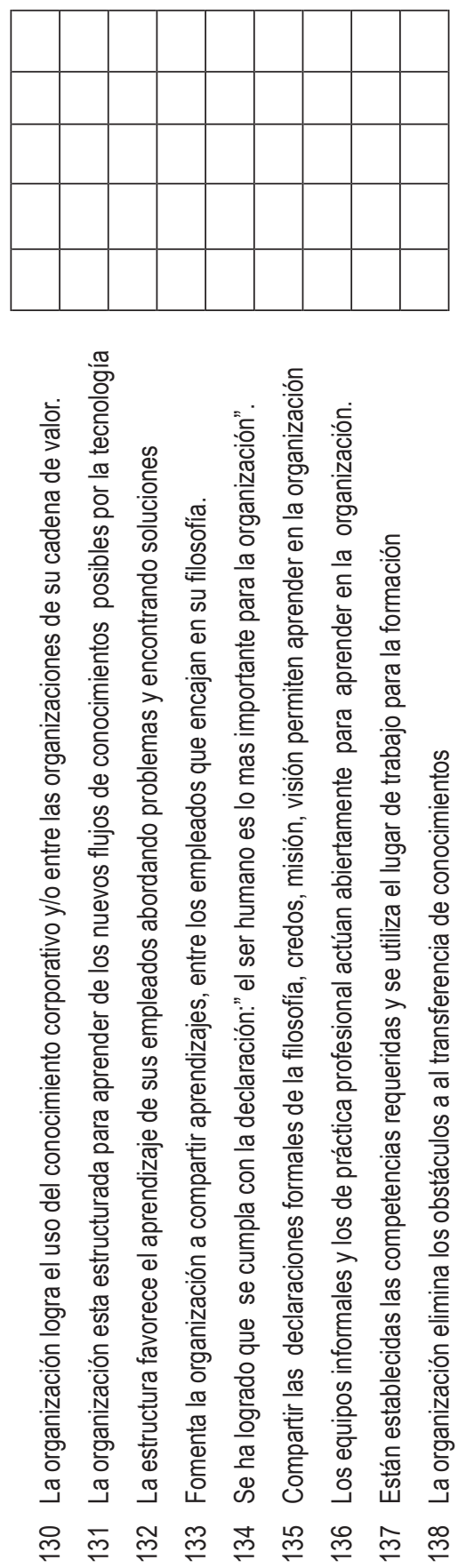


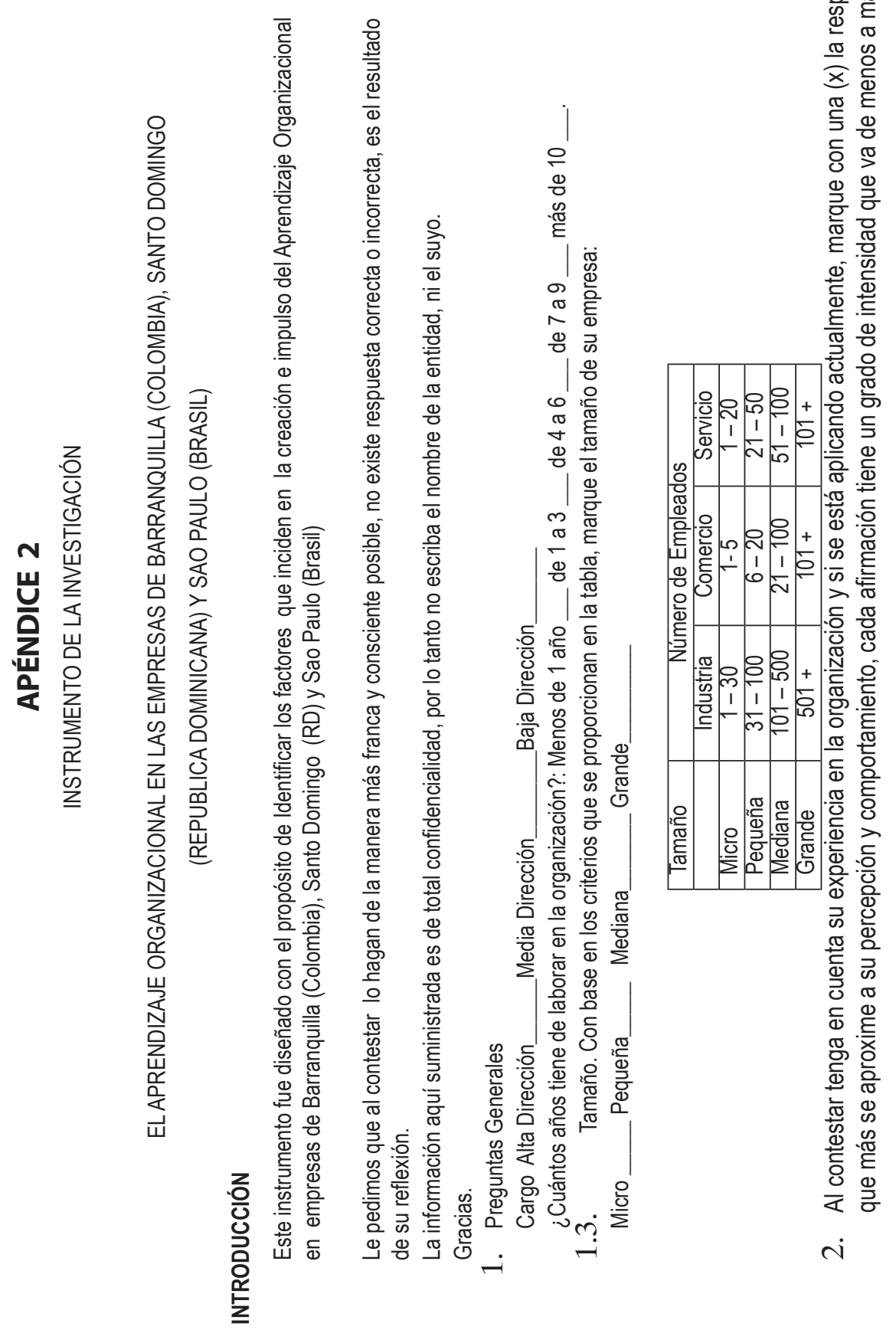

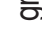
$\Phi$

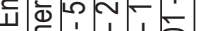
年 


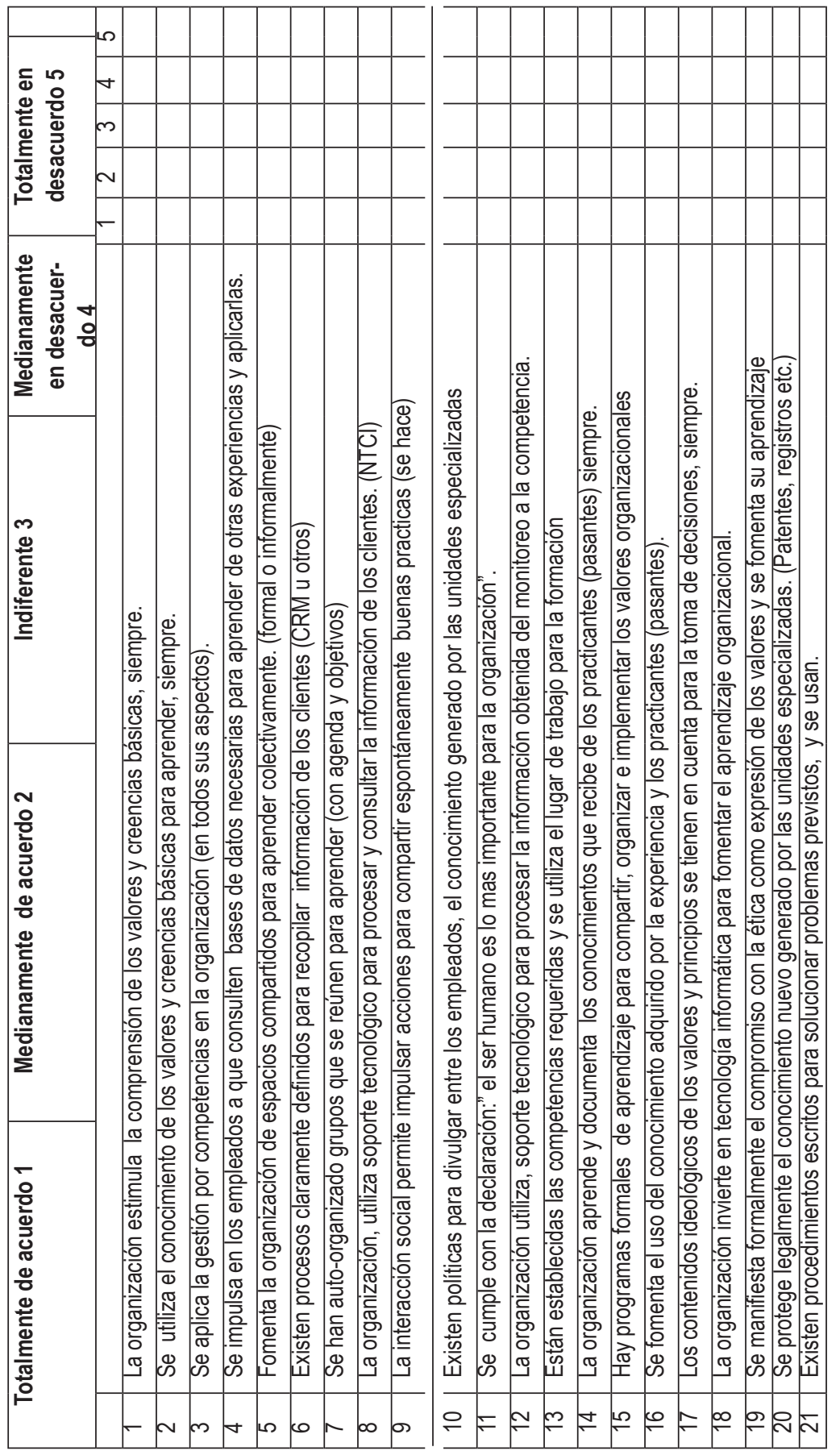




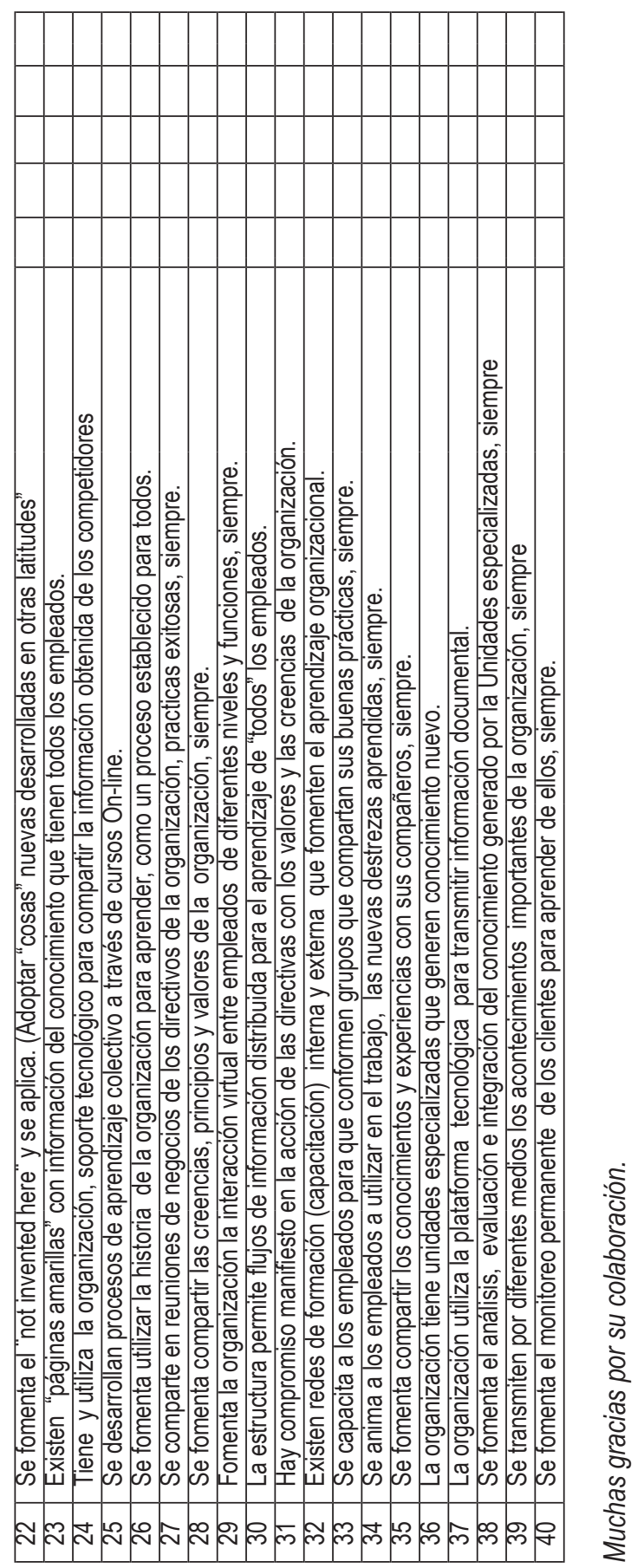

